\title{
Mathematics Model of the Question of Micro-pits Image with Direction-uniformity Distribution and Area Scrambling Micro-pits Image Design Methods Based on Regular Graphics Arrangement
}

\author{
Hongcai Wang ${ }^{1, ~ a}$ and Yang Wang ${ }^{2, b}$ \\ ${ }^{1}$ Key Laboratory of Mechanics in Advanced Manufacturing, Institute of Mechanics, Chinese \\ Academy of Sciences, Beijing, 100190, China \\ ${ }^{2}$ School of Mechatronics Engineering, Harbin Institute of Technology, Harbin, Heilongjiang, 150001, \\ China \\ ahcwang@imech.ac.cn, ${ }^{b}$ wyyh@hit.edu.cn
}

Keywords: Mathematics model, Surface roughness evaluation, Image design methods, Uniformity.

\begin{abstract}
Mathematical model including design variables, constraint conditions and target functions is raised on the basis of problem description. Using the mathematical model, area scrambling micro-pits image design methods are presented. The methods can generate square area scrambling micro-pits image based on square-aligned-array arrangement, square area scrambling micro-pits image based on square-staggered-array arrangement, and area scrambling micro-pits image based on equilateral hexagon-array arrangement. The uniformity of the last one is the best.
\end{abstract}

\section{Introduction}

Problem description of isotropic distribution of micro-pits is how to design the positions of micro-pits whose number is appointed and whose positions should be no overlap in an appointed plane to make $S R a_{d c v}$ the approximate minimum. $S R a_{d c v}$ referred to here is the coefficient of variation of directional surface arithmetic mean deviation in assessed area ${ }^{[1]}$. This is a quite complex global optimization problem. The number of possible micro-pits images is very large, but is limited afer all. So there must be an optimal image. In order to simplify the complex problem and acquire a micro-pits image with approximate isotropic distribution, this paper puts forward micro-pits image design methods based on regular graphics arrangement and area scrambling.

\section{Mathematical model}

Design variables This paper adopts image evaluation method and uses gray image to represent 3D surface. Each pixel represents the surface of $2 \times 2 \mu \mathrm{m}^{2}$ square area. Pixel gray value represents the mean value of the height of the small area. Each gray level represents the height of $0.1 \mu \mathrm{m}$. The position of the pixel in the image represents the 2D coordinates of the small area.

The sketch of a micro-pit is shown in Fig.1. A micro-pit consists of a ring-shaped lug and the pit. In the sectional view, the lug is simulated by the first half cycle of a sine wave, and the pit is simulated by the next half cycle of a sine wave. The external diameter of the micro-pit is $150 \mu \mathrm{m}$ and internal diameter ( the external diameter of the pit) is $100 \mu \mathrm{m}$. The lug height of the micro-pit is $5 \mu \mathrm{m}$. The depth of the micro-pit is $20 \mu \mathrm{m}$. In the platform of the micro-pit, the pixel gray value of the plane part is 200 ( This represents the value of the height is $20 \mu \mathrm{m}$ ). The pixel gray value of the top of the boss is 250 ( This represents the value of the height is $25 \mu \mathrm{m}$ ). The pixel gray value of the bottom of the pit is 0 ( This represents the value of the height is $0 \mu \mathrm{m}$ ). The lighter the colour, the higher the height.

The design coordinate system of micro-pits image is shown in Fig.2. The coordinate unit in the figure is the number of pixels (All the length units without indicating thereinafter are the number of pixels). $1501 \times 1501$ area repesents the size of the micro-pits to generate. The disk in the middle whose diameter is 1175 represents assessed area. The disk whose diameter is 75 represents the size of a micro-pit. 
In order to compare easily, all the sizes of the micro-pits images generated are $1501 \times 1501$. The number of the aligned micro-pits is about 196. The percentage of the cover ( the ratio of the total area of the micro-pits to the image area) is about $38.5 \%$. Suppose the 2D coordinates are $\left(x_{i}, y_{i}\right)$, design variables are represented by $1 \mathrm{D}$ vector $\left[\mathrm{x}_{1}, \mathrm{y}_{1}, \mathrm{x}_{2}, \mathrm{y}_{2} \cdots \mathrm{x}_{391}, \mathrm{y}_{392}\right]$ including 392 elements.

Constraint conditions According to the requirements that the micro-pits should be all in the area of $1501 \times 1501$ and their positions should be no overlap, constraint conditions are as follows:

$$
\begin{aligned}
& 38 \leq x_{i} \leq 1464 \quad i=1,2 \cdots 196 \\
& 38 \leq y_{i} \leq 1464 \quad i=1,2 \cdots 196 \\
& \sqrt{\left(x_{j}-x_{k}\right)^{2}+\left(y_{j}-y_{k}\right)^{2}} \geq 75 \quad j, k=1,2 \cdots 196, \mathrm{j} \neq \mathrm{k}
\end{aligned}
$$

When design a specific micro-pits image, the positions of the micro-pits are often determined by additive constraint conditions. The more the constraint conditions, the less the number of micro-pits images, and the worse the effect.

Target functions After generating the micro-pits image, $S R a$, a height amplitudes based on area-direction character, can be calculated by rotating and intercepting accessed area (the disk whose diameter is 1175). $S R a(j)$ can be acquired by dividing 180 degrees into 200 equal segments $(j$ $=1,2 \ldots 200)$.

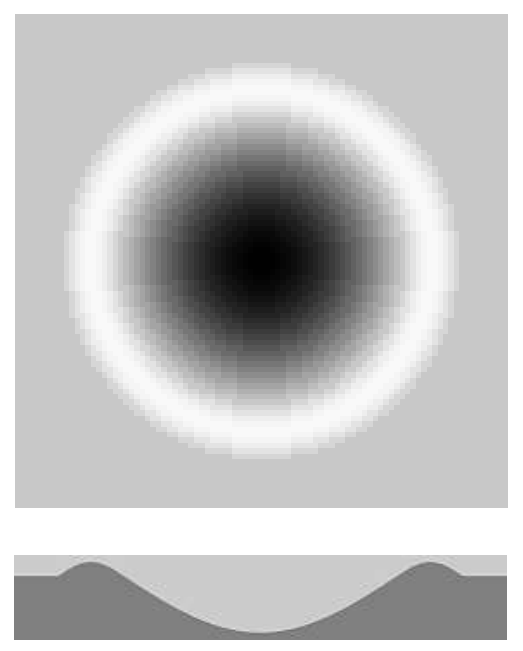

Fig.1 Sketch of a micro-pit

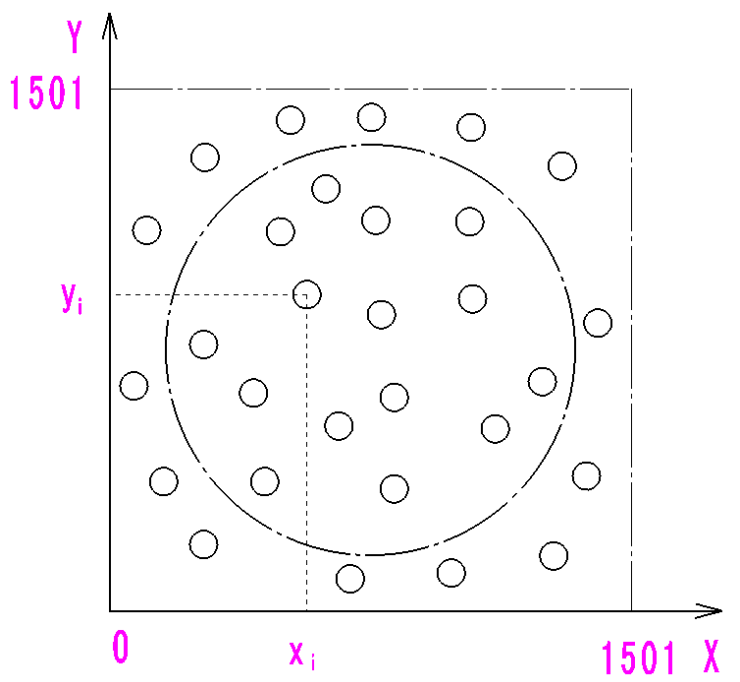

Fig.2 Design coordinate system of micro-pits images

Accessed area consists of 1175 horizontal close aligned rectangles, and each width of the rectangle is a single pixel.

$$
\operatorname{SRa}(j)=\frac{1}{1175} \sum_{i=1}^{1175} R a_{i}(j) \quad j=1,2 \cdots 200
$$

The directional accessed indexes are calculated as follows:

$$
\begin{aligned}
& S R a_{d \min }=\min [\operatorname{SRa}(j)] \quad j=1,2 \cdots 200 \\
& S R a_{d \max }=\max [S R a(j)] \quad j=1,2 \cdots 200 \\
& S R a_{d r a n g e}=S R a_{d \max }-S R a_{d \min } \\
& S R a_{d \text { mean }}=\frac{1}{200} \sum_{j=1}^{200} S R a(j) \quad j=1,2 \cdots 200 \\
& S R a_{\text {dstd }}=\sqrt{\frac{1}{200} \sum_{j=1}^{200} S R a(j)^{2}-S R a_{\text {dmean }}^{2}} \quad j=1,2 \cdots 200
\end{aligned}
$$




$$
S R a_{d c v}=\frac{S R a_{d s t d}}{S R a_{d m e a n}}
$$

In these formula, $S R a_{d \min }$ is the minimum directional surface arithmetic mean deviation.

$S R a_{d \max }$ is the maximum directional surface arithmetic mean deviation.

$S R a_{\text {drange }}$ is the range of directional surface arithmetic mean deviation.

$S R a_{\text {dmean }}$ is the mean deviation of directional surface arithmetic mean deviation.

$S R a_{d s t d}$ is the standard deviation of directional surface arithmetic mean deviation.

$S R a_{d c v}$ is the coefficient of variation of directional surface arithmetic mean deviation.

$S R a_{d c v}$ is the target funtion to optimize. The requirement is minimized.

\section{Area scrambling micro-pits image design methods based on regular graphics arrangement}

First step, the plane is divided into seamless equal segments according to a certain regular graphics, and the micro-pit is aligned in the middle of the regular graphics to generate uniform and regular micro-pits image. This image ensures the area uniformity of the micro-pits distribution.

Second step, the optimization target is to make $S R a_{d c v}$ of the image minimum. Moving the micro-pit ramdomly in the limited area of the regular graphics to acquire micro-pits image of approximate isotropic distribution.

There are three possible regular graphics arrays including square-aligned-array arrangement, square-staggered-array arrangement, and equilateral-hexagon-array arrangement. The optimization method LDSE(Low Dimensional Simplex Evolution Algorithms) is thereinafter adopted ${ }^{[2]}$.

Square area scrambling micro-pits image based on square-aligned-array arrangement. $14 \times$ 14 micro-pits are aligned by square array on the image whose size is $3 \times 3 \mathrm{~mm}^{2}$. The distance of the micro-pits is $200 \mu \mathrm{m}$. These micro-pits are placed in the middle. The center of each micro-pit is changed in the square of $50 \times 50 \mu \mathrm{m}^{2}$. The optimization target is to make $S R a_{d c v}$ minimum. After repeated searches, the approximate isotropic distribution of micro-pits image is acquireed. The result is shown in Fig.3. Its graph of $S R a$ is shown in Fig.4. Its directional accessed indexes of $S R a$ are shown in the data at row 1 in Table 1.

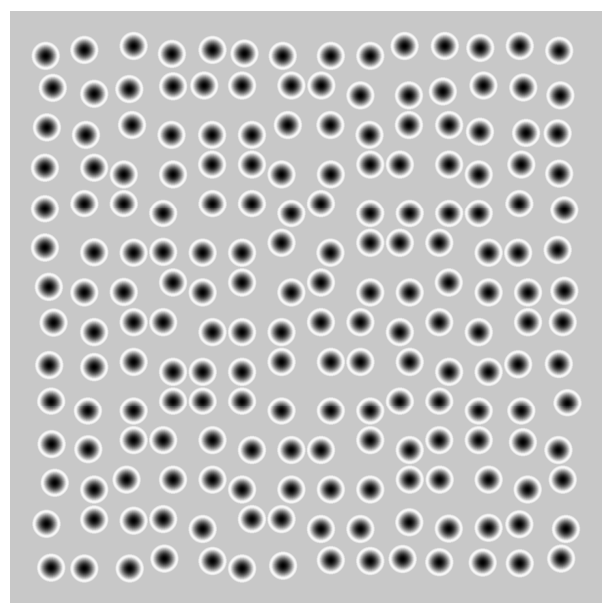

Fig.3 Image based on square- aligned-array and area scrambling of micro-pits

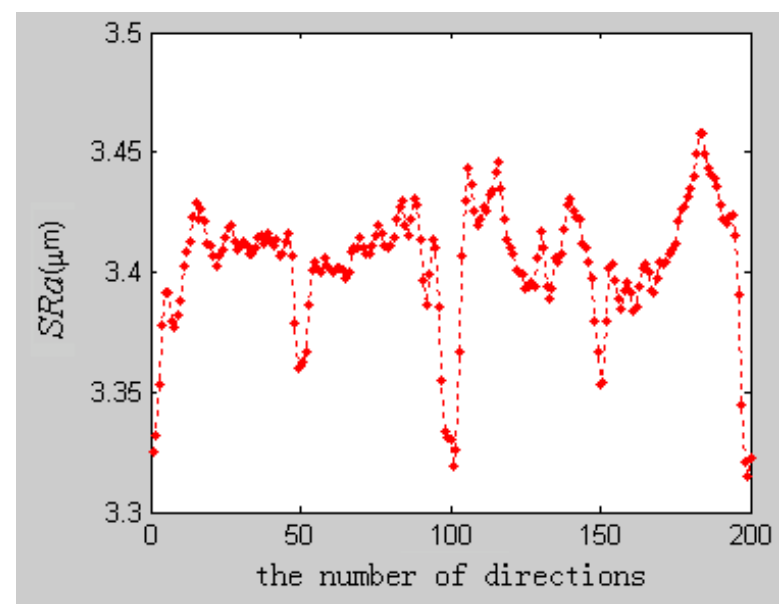

Fig.4 Graph of $S R a$ of the image based on square-aligned-array and area scrambling of micro-pits

Square area scrambling micro-pits image based on square-staggered-array arrangement. $S R a$ of the micro-pits image based on square-aligned-array have two minimal values in the directions of 90 degree and 180 degree, which cause the value of $S R a_{d c v}$ too big. If initial micro-pits are aligned in a square-staggered-array, the minimal value of $S R a$ in the direction of 90 degree can be eliminated, and $S R a_{d c v}$ of $S R a$ can be decreased further. 
$14 \times 14$ micro-pits are aligned by square-staggered-array on the image whose size is $3 \times 3 \mathrm{~mm}^{2}$. The distance of the micro-pits is $200 \mu \mathrm{m}$. These micro-pits are placed in the middle. The center of each micro-pit is changed in the square of $50 \times 50 \mu \mathrm{m}^{2}$. The optimization target is to make $S R a_{d c v}$ minimum. After repeated searches, the approximate isotropic distribution of micro-pits image is acquireed. The result is shown in Fig.5. Its graph of $S R a$ is shown in Fig.6. Its directional accessed indexes of $S R a$ are shown in the data at row 2 in Table 1.

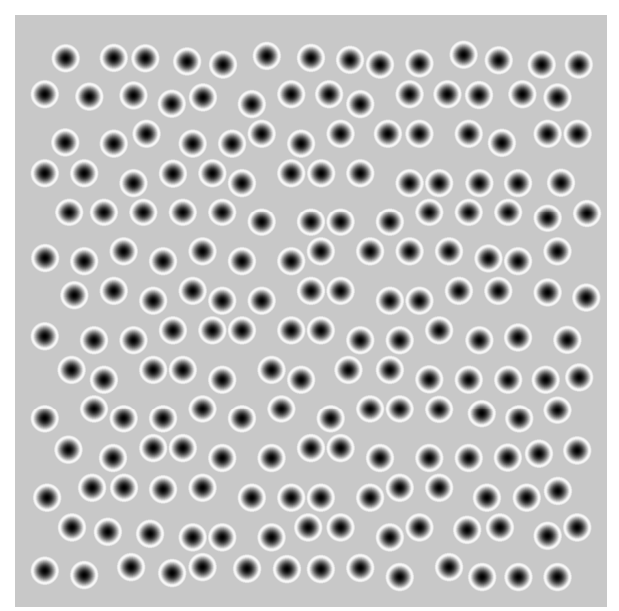

Fig.5 Image based on square-staggered-array and area scrambling of micro-pits

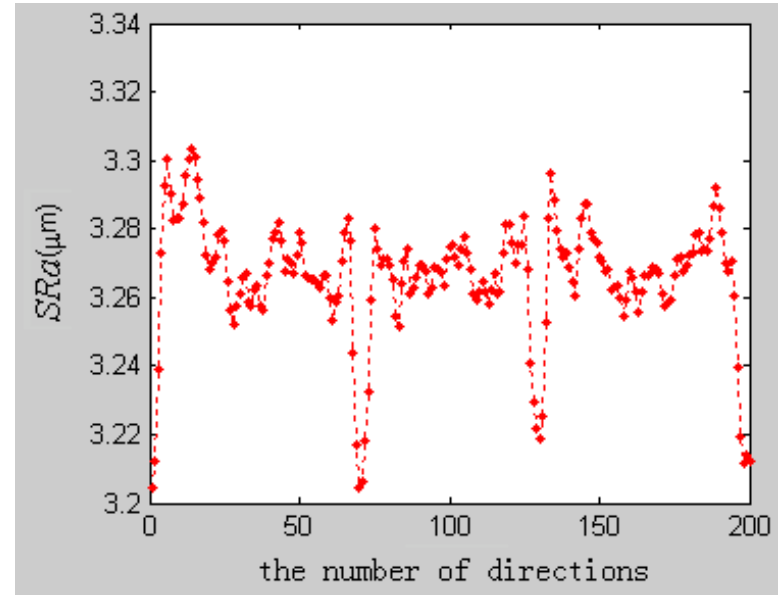

Fig. 6 Graph of $S R a$ of the image based on square-staggered-array and area scrambling of micro-pits

Area scrambling micro-pits image based on equilateral hexagon-array arrangement. The regular micro-pits image based on equilateral hexagon-array arrangement can ensure uniform distribution in three directions. However, the regular micro-pits image based on square-staggeredarray arrangement can only ensure uniform distribution in two directions. $S R a_{d c v}$ of the area scrambling micro-pits image based on equilateral hexagon-array arrangement may smaller.

$13 \times 15$ micro-pits are aligned by equilateral-hexagon-array on the image whose size is $3 \times 3 \mathrm{~mm}^{2}$. Horizontal distance of the micro-pits is $216 \mu \mathrm{m}$ and vertical distance of the micro-pits is $186 \mu \mathrm{m}$. These micro-pits are placed in the middle. The center of each micro-pit is changed in the equilateral hexagon whose side length is $38 \mu \mathrm{m}$. The optimization target is to make $S R a_{d c v}$ minimum. After repeated searches, the approximate isotropic distribution of micro-pits image is acquired. The result is shown in Fig.7. Its graph of $S R a$ is shown in Fig.8. Its directional accessed indexes of $S R a$ are shown in the data at row 3 in Table 1.

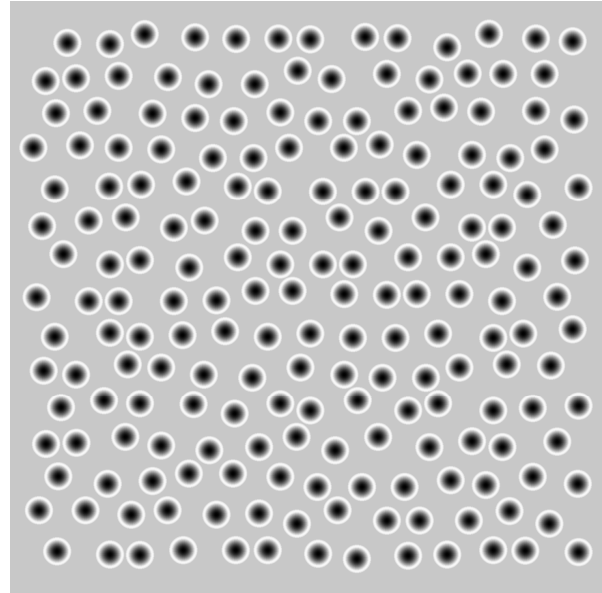

Fig.7 Image based on equilateral hexagon-array and area scrambling of micro-pits

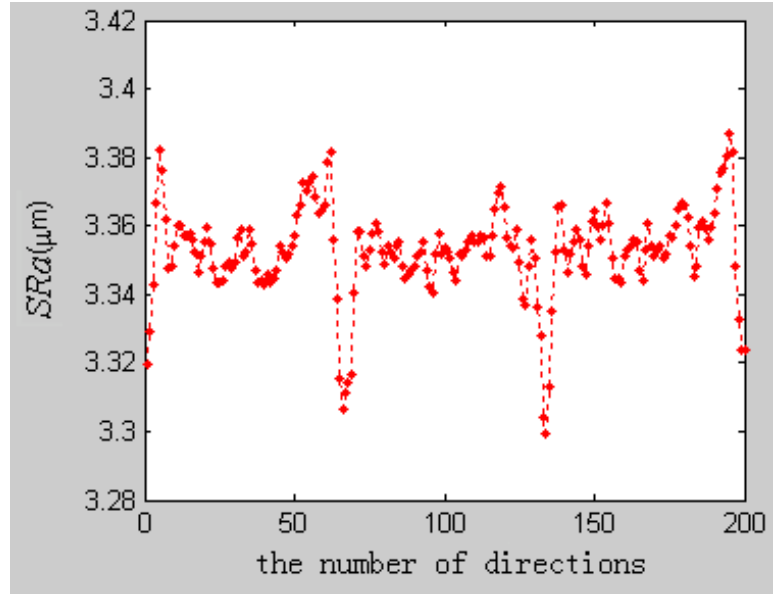

Fig. 8 Graph of $S R a$ of the image based on equilateral hexagon-array and area scrambling of micro-pits 
Table1 Assessed direction indexes of $S R a$ of the micro-pits images

\begin{tabular}{cccccc}
\hline$S R a_{\text {dmin }}(\mu \mathrm{m})$ & $S R a_{\text {dmax }}(\mu \mathrm{m})$ & $S R a_{\text {drange }}(\mu \mathrm{m})$ & $S R a_{\text {dmean }}(\mu \mathrm{m})$ & $S R a_{\text {dstd }}(\mu \mathrm{m})$ & $S R a_{d c v}(\%)$ \\
\hline 3.3148 & 3.4579 & 0.1431 & 3.4038 & 0.0264 & 0.7761 \\
3.2043 & 3.3032 & 0.0989 & 3.2662 & 0.0178 & 0.5463 \\
3.2996 & 3.3871 & 0.0875 & 3.3525 & 0.0135 & 0.4036 \\
\hline
\end{tabular}

\section{Conclusions}

The biggest advantage of the area scrambling micro-pits image design methods based on regular graphics arrangement is convenient for optimization. Distributing micro-pits regularly by area can ensure the area uniformity of micro-pits distribution. Area scrambling micro-pits and the optimization of the distribution can ensure approximate isotropic of micro-pits distribution.

As shown in Table 1, the uniform effect of three micro-pits images are all good. In comparison, the uniform effect of the area scrambling micro-pits image based on equilateral hexagon-array arrangement is the best.

\section{References}

[1] Hongcai Wang, Yang Wang. The Height Amplitudes of Surface Roughness based on Area-direction Character and Relative Assessed Indexes[J]. Advanced Material Research, 482-484(2012): 1150-1154.

[2] Changtong Luo. Low dimensional simplex evolution algorithms and their applications[D]. Jilin University, 2007(in Chinese). 
Mechatronics and Intelligent Materials III

10.4028/www.scientific.net/AMR.706-708

Mathematics Model of the Question of Micro-Pits Image with Direction-Uniformity Distribution and Area Scrambling Micro-Pits Image Design Methods Based on Regular Graphics Arrangement

10.4028/www.scientific.net/AMR.706-708.407 